\title{
Semiconductor materials and radiation detection
}

\author{
Alan Owens \\ Science Payload and Advanced Concepts Office, \\ SCI-AT, ESA/ESTEC, Postbus 299, 2200AG Noordwijk, The Netherlands \\ e-mail: aowens@rssd.esa.int
}

While $\mathrm{Si}$ and Ge have become detection standards for X- and gamma-ray spectroscopy in the laboratory, their use for an increasing range of applications is becoming marginalized by one or more of their physical limitations; namely the need for ancillary cooling systems or bulky cryogenics, their modest stopping powers and radiation intolerance. Wide band gap compounds offer the ability to operate in a range of chemical, thermal and radiation environments, whilst still maintaining sub-keV spectral resolution at X-ray wavelengths. In addition, these materials encompass such a wide range of physical properties that it is technically feasible to engineer materials to specific applications. However, while compound materials are used routinely in the optical and infrared wavebands, their development at hard X-and gamma-ray wavelengths has been plagued by material and fabrication problems. In this paper we present an overview of suitable materials and review the current progress in producing $\mathrm{X}$ - and gamma-ray radiation detectors.

Keywords: compound semiconductors; radiation detectors; X-rays; gammarays.

\section{Introduction}

Semiconductor materials can generally be grouped into 2 categories. These are the elemental semiconductors, Si and Ge, which are derived from elements in Group IV of the periodic table and compound semiconductors - the most common of which are derived from groups III and V (e.g., GaAs, InP) and groups II and VI (e.g., CdTe) of the periodic table. Compound semiconductors are so useful because of the shear range of compounds available. This is illustrated in Table 1 in which we list the semiconductors available as a function of group and band gap energy. In addition to binary materials (such as GaAs or InP), most compounds are also soluble within each other, making it possible to synthesize ternary (e.g., AlGaAs, HgCdTe) quaternary (e.g., InGaAsP, InGaAlP) and higher order solutions, simply by alloying binary compounds together. The basic problem in producing detector grade material is the difficulty of growing chemically pure and structurally perfect crystals with exact stoichiometry.

For radiation detection, compound semiconductors have a number of distinct advantages over their elemental counterparts, arising from the wide range of stopping powers and band gaps available. For example, band gap energies range from $\sim 1.35$ to $\sim 2.6 \mathrm{eV}$ and average atomic numbers from $\sim 30$ to $\sim 90$ (see Table 1). This suggests that it may be possible to mix and match available band gaps and stopping powers, which in turn implies that materials can be targeted for specific applications in 
radiation detection. For example, within a given target spectral resolution and detection efficiency, it is usually possible to select from a range of stopping powers.

Table 1. Compound semiconductor materials listed by Group and in order of increasing band gap energy ranging from the near IR to XUV wavelengths. Materials subscripted with a "*"are those for which spectroscopic measurements (i.e., $E / \Delta E>1$ ) have been made at X-ray wavelengths. Here, $\Delta E$ is the FWHM energy resolution at energy $E$. The compounds subscripted with a "++" are those which have shown some response to radiation.

\begin{tabular}{|c|c|c|c|c|c|c|c|}
\hline $\begin{array}{l}\text { Band-gap } \\
\text { energy (eV) }\end{array}$ & $\begin{array}{c}\text { Elemental } \\
\text { Group IVB }\end{array}$ & $\begin{array}{c}\text { Binary IV-IV } \\
\text { Compounds }\end{array}$ & $\begin{array}{l}\text { Binary III-V } \\
\text { Compounds }\end{array}$ & $\begin{array}{l}\text { Binary II-VI } \\
\text { Compounds }\end{array}$ & $\begin{array}{c}\text { Binary IV-VI } \\
\text { Compounds }\end{array}$ & $\begin{array}{c}\text { Binary n-VIIB } \\
\text { Compounds }\end{array}$ & Ternary Compounds \\
\hline $0.00-0.25$ & Sn & & ${ }^{++} \mathrm{InSb}$ & $\mathrm{HgTe}$ & & & $\mathrm{HgCdTe}$ \\
\hline $0.25-0.50$ & & & InAs & $\mathrm{HgSe}$ & $\begin{array}{c}\text { PbSe, } \\
\mathrm{PbS}, \mathrm{PbTe}\end{array}$ & & \\
\hline $0.50-0.75$ & ${ }^{*} \mathrm{Ge}$ & & $\mathrm{GaSb}$ & & & & InGaAs \\
\hline $0.75-1.00$ & & SiGe & & & & & \\
\hline $1.00-1.25$ & ${ }^{*} \mathrm{Si}$ & & & & $\mathrm{SnS}$ & & \\
\hline $1.25-1.50$ & & & ${ }^{*} \mathrm{GaAs}$, ${ }^{*}$ InP & ${ }^{2} \mathrm{CdTe}$ & & & AlInAs \\
\hline $1.50-1.75$ & & & $\mathrm{AlSb}$ & ${ }^{*} \mathrm{CdSe}$ & & & $\mathrm{AlGaAs}$ \\
\hline $1.75-2.00$ & & & $\mathrm{BP}, \mathrm{InN}$ & & & ${ }^{+1+} \mathrm{BiI}_{3}$ & ${ }^{*} \mathrm{CdZnTe},{ }^{*} \mathrm{CdZnSe}$, InAll \\
\hline $2.00-2.25$ & & ${ }^{*} \mathrm{SiC}$ & AlAs & $\mathrm{HgS}$ & & ${ }^{*} \mathrm{HgI}_{2}$ & ${ }^{*} \mathrm{CdMnTe}$ \\
\hline $2.25-2.50$ & & & ${ }^{+} \mathrm{GaP}, \mathrm{AlP}$ & $\mathrm{ZnTe}, \mathrm{CdS}$ & & ${ }^{2} \mathrm{PbI} \mathrm{I}_{2}$ & ${ }^{*} \mathrm{TlBrI}, \mathrm{InAlP}, \mathrm{TlPbI}_{3}$ \\
\hline $2.50-2.75$ & & & & ${ }^{++} \mathrm{ZnSe}$ & & ${ }^{*} \mathrm{TlBr}$ & $\mathrm{HgBrI}$ \\
\hline $2.75-3.00$ & & & & $\mathrm{MnSe}$ & & & \\
\hline $3.00-3.25$ & & & & $\mathrm{MnTe}$ & & & \\
\hline $3.25-3.50$ & & & $\mathrm{GaN}$ & $\mathrm{MgTe}, \mathrm{MnS}$ & & & \\
\hline $3.50-3.75$ & & & & $\mathrm{MgSe}, \mathrm{ZnS}$ & & & \\
\hline $3.75-4.00$ & & & & & & & \\
\hline $4.00-4.25$ & & & & & & & \\
\hline $4.25-4.50$ & & & & $\mathrm{MgS}$ & & & \\
\hline $4.50-4.75$ & & & & & & & \\
\hline $4.75-5.00$ & & & & & & & \\
\hline $5.00-5.25$ & & & & & & & \\
\hline $5.25-5.50$ & ${ }^{++} \mathrm{C}$ & & & & & & \\
\hline $5.50-5.75$ & & & & & & & \\
\hline $5.75-6.00$ & & & $\mathrm{BN}$ & & & & \\
\hline $6.00-6.25$ & & & AlN & & & & \\
\hline $6.25-6.50$ & & & & & & & \\
\hline $6.50-6.75$ & & & & & & & \\
\hline $6.75-7.00$ & & & & & & & \\
\hline
\end{tabular}

Choosing materials with the largest stopping powers enables thinner detectors to be produced with resulting benefits in radiation tolerance (which is a bulk effect) and lower leakage currents. Alternatively, choosing smaller stopping powers will increase scattering efficiency, which is a requirement for polarimetry measurements. These compounds can also be tailored towards tissue equivalence. By careful choice of both band gap and stopping power it is possible to fabricate detectors with a very wide dynamic range but sub-keV energy resolution in the soft X-ray band and tens of $\mathrm{keV}$ in the gamma-ray band. For space research this is particularly attractive, since it has mass and cost benefits in spacecraft design in that by using denser materials smaller detection systems can be fabricated without losing spectral acuity. Finally, unlike Si and Ge whose electronic and chemical properties are "fixed", those of compound semi-conductors can be modified by band gap engineering. Specifically, the energy gap between the valence and conduction states is defined by the inter-atomic distance and the electronegativity of the component atoms. Changing the composition of an alloy, changes its average lattice constant to reflect the different atomic bonding radii of the constituent elements. This changes the band gap energy, which in turn alters the properties of the material. A specific example of how this is useful for radiation detector applications is $\mathrm{Cd}_{(1-\mathrm{x})} \mathrm{Zn}_{\mathrm{x}}$ Te in which adding a few percent $\mathrm{Zn}$ to $\mathrm{CdTe}$ not 
only increases the band gap energy, but also increases the energy of defect formation and its mechanical strength. Consequently leakage currents and resistivities are correspondingly higher.

\section{Radiation detection using compound semiconductors}

All semiconductor detectors operate as solid-state ionization chambers. In figure 1 we illustrate a simple planar detector geometry. Ionizing radiation absorbed in the sensitive volume excites electron-hole pairs in direct proportion to the energy deposited (i.e., $n_{\mathrm{o}}=E_{\mathrm{o}} / \varepsilon$, where $n$ is the number of electron-hole pairs generated, $E_{\mathrm{o}}$ is the energy deposited and $\varepsilon$ is the average energy consumed to create an electronhole

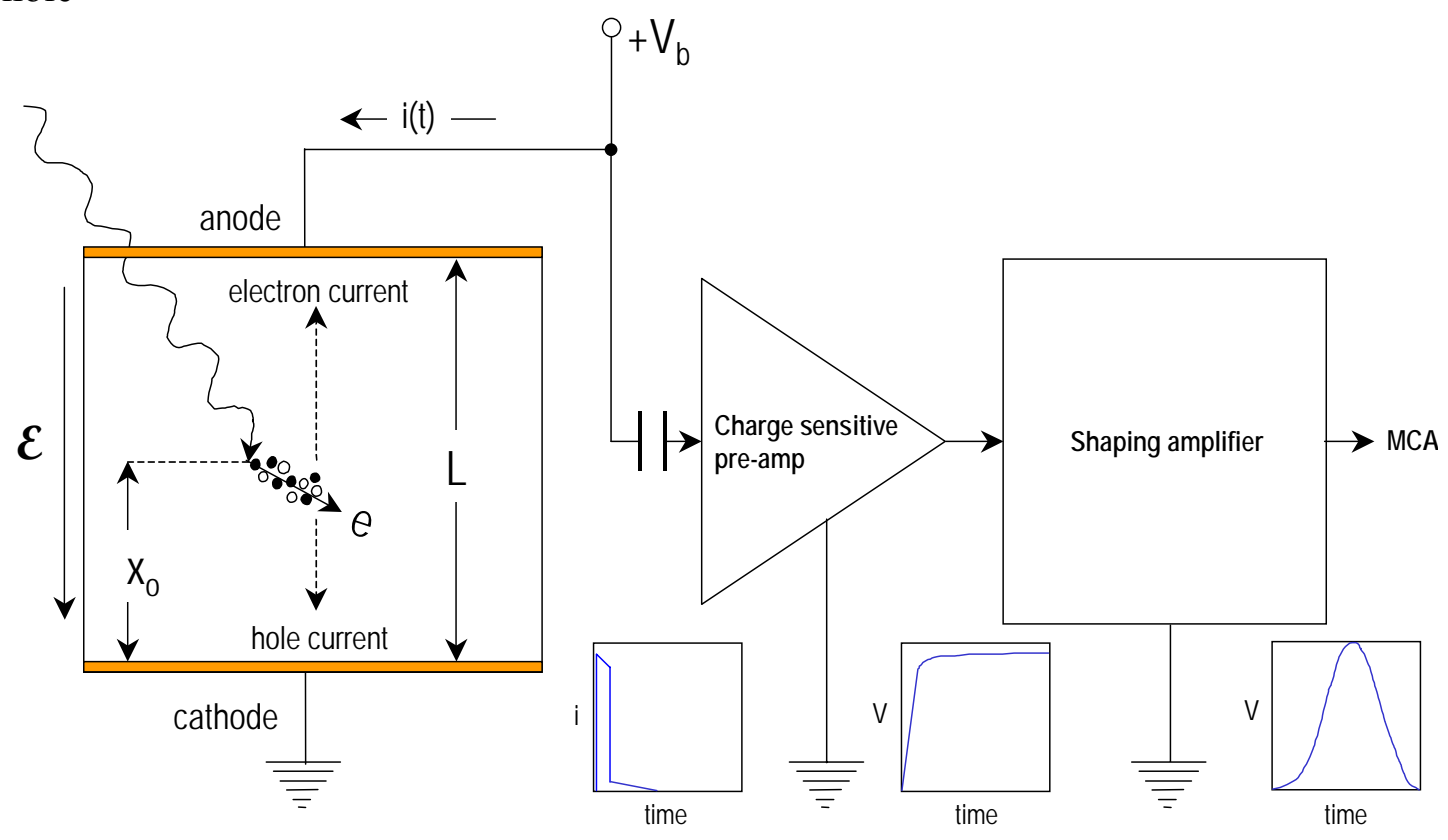

Figure 1. Left: Schematic of a simple planar detection system. For completeness we also show the signal chain and the evolution of currents and voltages. Ionizing radiation absorbed in the sensitive volume generates electron-hole pairs in direct proportion to the energy deposited. These are subsequently swept towards the appropriate electrode by the electric field induced by the bias voltage $\mathrm{V}_{\mathrm{b}}$.

pair). Applying an electric field across the detector causes the liberated carriers to drift towards the anode and cathode with drift velocities $v_{\mathrm{e}}$ and $v_{\mathrm{h}}$, creating an induced charge on the electrodes and by current continuity into the electronics. The induced charge $Q_{\text {ind }}$ is given by the Shockley-Ramo theorem (Shockley 1938; Ramo 1939) which makes use of the concept of a weighting potential. The weighting potential is defined as the potential that would exist in the detector with the collecting electrode held at unit potential, while holding all other electrodes at zero potential. The theorem states that regardless of the presence of space charge, the change in the induced charge $\Delta Q_{\text {ind }}$ and the current $i$ at an electrode caused by a charge $q$ moving from $x_{\mathrm{i}}$ to $x_{\mathrm{f}}$ are given by

$\Delta Q_{\text {ind }}=\int_{x \mathrm{i}}^{x \mathrm{f}} q E_{\mathrm{w}}(x) \cdot d x=-q\left[V_{\mathrm{w}}\left(x_{\mathrm{f}}\right)-V_{w}\left(x_{\mathrm{i}}\right)\right]$ and $\quad i=\frac{d Q}{d t}=q v \cdot E_{\mathrm{w}}$, 
where $V_{\mathrm{w}}(x)$ and $E_{\mathrm{w}}(x)$ is the weighting potential and field at position $x$. The total induced charge will actually be the sum of the induced charges due to the electron and holes. The integration of these charges gives the total charge, $Q$. In the absence of trapping, $Q=Q_{0}=-q n_{0}$, the original charge created, which in turn is proportional to the energy of the incident photon. However, in any semiconductor some density of electron and hole traps are always present and these result in a loss of carriers and therefore charge at the electrodes. For compound semiconductors, crystal growth practices lead to a much higher density of traps than in the elemental semiconductors and hence shorter lifetimes. For example, in CdZnTe, typical lifetimes are $\tau_{\mathrm{e}}=3 \times 10^{-6}$ sec and $\tau_{\mathrm{h}}=5 \times 10^{-8}$ sec. Because the hole lifetime is much shorter than the hole transit time the induced charge is significantly reduced and is now dependent upon the depth of interaction, $\mathrm{x}_{0}$. In the case of a uniform electric field and negligible de-trapping, the fraction of charge that is induced at the electrodes is best described by the charge collection efficiency (CCE), which is given by the Hecht equation (Hecht 1932)

$$
C C E=\frac{Q}{Q_{\mathrm{o}}}=\frac{\lambda_{\mathrm{e}}}{L}\left[1-\exp \left(-\frac{\left(L-x_{\mathrm{o}}\right)}{\lambda_{\mathrm{e}}}\right)\right]+\frac{\lambda_{\mathrm{h}}}{L}\left[1-\exp \left(-\frac{x_{\mathrm{o}}}{\lambda_{\mathrm{h}}}\right)\right],
$$

where $L$ is the detector thickness, $x_{\mathrm{o}}$ is the distance from the cathode to the point of charge creation and $\lambda_{\mathrm{e}}$ and $\lambda_{\mathrm{h}}$ are the carrier drift lengths in the applied electric field, $\mathcal{E}$, given by, $\lambda_{\mathrm{e}}=\mu_{\mathrm{e}} \tau_{\mathrm{e}} \mathcal{E}$ and $\lambda_{\mathrm{h}}=\mu_{\mathrm{h}} \tau_{\mathrm{h}} \mathcal{E}$. Here, $\mu_{\mathrm{e}}$ and $\mu_{\mathrm{h}}$ are the electron and hole mobilities and $\tau_{\mathrm{e}}$ and $\tau_{\mathrm{h}}$ are the corresponding lifetimes. It follows from eqn. (2) that the CCE depends not only on $\lambda_{\mathrm{e}}$ and $\lambda_{\mathrm{h}}$, but also on the location where the charge was created. Since the interaction points of incident photons is essentially random at intermediate and high energies, being weighted by the classical exponential absorption law, the width of the peak in the energy spectrum broadens to a degree governed by the ratios $\lambda_{\mathrm{e}} / L$ and $\lambda_{\mathrm{h}} / L$.

Table 2. Room temperature properties of wide band gap compound semiconductor materials suitable for hard X- and $\gamma$-ray detectors. The abbreviations are: $\mathrm{C}=$ Czochralski, $\mathrm{CVD}=$ Chemical Vapour Deposition, LEC = Liquid Encapsulated Czochralski, THM = Traveller Heater Method, BM = Bridgman Method, HPB = High Pressure Bridgman and VA=Vertical Ampoule Method.

\begin{tabular}{|l|llcccccc|}
\hline Material & $\begin{array}{l}\text { Growth } \\
\text { method }\end{array}$ & $\begin{array}{c}\text { Atomic } \\
\text { Number }\end{array}$ & $\begin{array}{c}\text { Density } \\
\left(\mathrm{g} / \mathrm{cm}^{3}\right)\end{array}$ & $\begin{array}{c}\text { Band } \\
\text { gap } \\
(\mathrm{eV})\end{array}$ & $\begin{array}{c}\text { Epair } \\
(\mathrm{eV})\end{array}$ & $\begin{array}{c}\text { Resist- } \\
\text { ivity } \\
(\Omega-\mathrm{cm})\end{array}$ & $\begin{array}{c}\mu \tau(\mathrm{e}) \\
\text { Product } \\
\left(\mathrm{cm}^{2} / \mathrm{V}\right)\end{array}$ & $\begin{array}{c}\mu \tau(\mathrm{h}) \\
\text { Product } \\
\left(\mathrm{cm}^{2} / \mathrm{V}\right)\end{array}$ \\
\hline \hline $\mathrm{Si}$ & $\mathrm{C}$ & 14 & 2.33 & 1.12 & 3.6 & $10^{4}$ & $4 \times 10^{-1}$ & $2 \times 10^{-1}$ \\
\hline $\mathrm{Ge}$ & $\mathrm{C}$ & 32 & 5.33 & 1.57 & 4.6 & 50 & 1 & 1 \\
\hline $4 \mathrm{H}-\mathrm{SiC}$ & $\mathrm{CVD}$ & 14,6 & 3.17 & 3.26 & 7.8 & $>10^{3}$ & $<5 \times 10^{-4}$ & $<8 \times 10^{-5}$ \\
\hline $\mathrm{GaAs}$ & $\mathrm{CVD}$ & 31,33 & 5.32 & 1.43 & 4.2 & $10^{11}$ & $10^{-2}$ & $5 \times 10^{-4}$ \\
\hline $\mathrm{InP}$ & $\mathrm{LEC}$ & 15,49 & 4.78 & 1.43 & 4.2 & $10^{6}$ & $5 \times 10^{-6}$ & $<2 \times 10^{-5}$ \\
\hline $\mathrm{CdTe}$ & $\mathrm{THM}$ & 48,52 & 6.2 & 1.44 & 4.4 & $10^{9}$ & $3 \times 10^{-3}$ & $2.6 \times 10^{-4}$ \\
\hline $\mathrm{Cd}_{0.9} \mathrm{Zn}_{0.1} \mathrm{Te}$ & $\mathrm{HPB}$ & $48,30,52$ & 5.78 & 1.57 & 4.6 & $10^{11}$ & $7 \times 10^{-3}$ & $9 \times 10^{-5}$ \\
\hline $\mathrm{PbI}_{2}$ & $\mathrm{BM}$ & 82,53 & 6.2 & $2.3-2.6$ & 4.9 & $10^{12}$ & $8 \times 10^{-6}$ & $9 \times 10^{-7}$ \\
\hline $\mathrm{HgI}_{2}$ & $\mathrm{VAM}$ & 80,53 & 6.4 & 2.13 & 4.2 & $10^{13}$ & $10^{-4}$ & $4 \times 10^{-5}$ \\
\hline $\mathrm{TlBr}^{13}$ & $\mathrm{BM}$ & 81,35 & 7.56 & 2.68 & 6.5 & $10^{12}$ & $3 \times 10^{-5}$ & $1.5 \times 10^{-6}$ \\
\hline
\end{tabular}


In characterizing materials, perhaps the most useful figure of merit is the mobilitylifetime product $(\mu \tau)$, which is directly related to the drift length. Poorer $\mu \tau$ products result in shorter drift lengths and therefore smaller $\lambda / L$, which in turn limit the maximum size and energy range of detectors. For the elemental semi-conductors $\mu \tau$ is of the order of unity $\mathrm{cm}^{2} \mathrm{~V}^{-1}$ for both electrons and holes, whereas for compound semiconductors it rarely reaches greater than a few times $10^{-4} \mathrm{~cm}^{2} \mathrm{~V}^{-1}$ for electrons and $10^{-5} \mathrm{~cm}^{2} \mathrm{~V}^{-1}$ for holes - and these figures get worse with increasing $Z$ (see Table 2 ). The cause can usually be traced to trapping centers caused by impurities, vacancies, structural irregularities (e.g., dislocations), or for the softer materials, plastic deformation caused by mechanical damage during fabrication.

\section{Spectral broadening in radiation detection systems}

In addition to broadening caused by poor charge collection, the width of the full energy peak, $\Delta E$, is also broadened by the statistics of carrier generation (Fano noise) and by electronic noise. For most compound semiconductors, however, these components are generally far less important than the noise due to incomplete charge collection, except for thin detectors where $\lambda_{\mathrm{e}} / L \gg 1$. The energy resolution, $\Delta E$, of the system is defined as the full width at half-maximum of the energy-loss distribution resulting from exposure to monoenergetic radiation. The width of the energy-loss spectrum, in turn, results from the convolution of the probability distributions of the various noise components. For an infinitely thin detector, three terms tend to dominate;

$$
\Delta E=f\left(\sigma_{\mathrm{F}}^{2}, \sigma_{\mathrm{e}}^{2}, \sigma_{\mathrm{c}}^{2}\right), \quad(\mathrm{keV})
$$

where, $\sigma_{\mathrm{F}}^{2}$ is the variance of the noise due to carrier generation or Fano noise, $\sigma_{\mathrm{e}}^{2}$ is the variance of the noise due to the leakage current and amplifier noise, and $\sigma_{\mathrm{c}}^{2}$ is the variance of the noise due to incomplete charge collection due to carrier trapping. The contribution due to Fano noise, $\Delta E_{\mathrm{F}}$, is calculated from

$$
\Delta E_{\mathrm{F}}=2.355 \sqrt{\sigma_{\mathrm{F}}^{2}}=2.355 \sqrt{F E \varepsilon}, \quad(\mathrm{keV})
$$

where $F$ is the Fano factor, $\varepsilon$ is the energy to create an electron-hole pair and $E$ is the incident energy. The electronic noise component, $\Delta E_{\mathrm{e}}$, is given by

$$
\Delta E_{\mathrm{e}}=2.355 \sqrt{I \tau \varepsilon^{2} A / e+\sigma_{\mathrm{a}}^{2}} \quad, \quad(\mathrm{keV})
$$

where $I$ is the leakage current, $e$ is the electronic charge, $A$ is a constant depending on the type of signal shaping, $\tau$ is the shaping time and $\sigma_{\mathrm{a}}^{2}$ is the variance of the amplifier shot noise which depends on the actual design and specifically the capacitive loading of the front end electronics. For the Gaussian shaping typically used in spectroscopy, $A=0.875$.

The functional form of $\Delta E_{\mathrm{c}}$ is much more difficult to predict, since it is intimately dependent on the trap density distribution as well as the charge diffusion and 
collection properties of the detector. For the case of $\lambda_{\mathrm{e}} \neq \lambda_{\mathrm{h}}$, which is invariably true for compound semiconductors, Iwanczyk et al. (1992) have arrived at the following analytic form for the relative broadening;

$$
\begin{aligned}
\left(\frac{\sigma_{\mathrm{c}}}{E}\right)^{2} & =\frac{2 \lambda_{\mathrm{e}}^{2} \lambda_{\mathrm{h}}^{2}}{L^{3}\left(\lambda_{\mathrm{e}}-\lambda_{\mathrm{h}}\right)}\left(e^{-L / \lambda_{\mathrm{e}}}-e^{-L / \lambda_{\mathrm{h}}}\right)-\frac{1}{L^{4}}\left[\lambda_{\mathrm{e}}^{2}\left(e^{-L / \lambda_{\mathrm{e}}}-1\right)+\lambda_{\mathrm{h}}^{2}\left(e^{-L / \lambda_{\mathrm{h}}}-1\right)\right]^{2} \\
& -\frac{\lambda_{\mathrm{e}}^{3}}{2 L^{3}}\left(e^{-2 L / \lambda_{\mathrm{e}}}-1\right)-\frac{\lambda_{\mathrm{h}}^{3}}{2 L^{3}}\left(e^{-2 L / \lambda_{\mathrm{h}}}-1\right),
\end{aligned}
$$

assuming a uniform electric field. However, while eqn. (6) can be used to calculate the width $\Delta E_{\mathrm{c}}$, the shape of the pulse height distribution can only be realistically evaluated by following the approach of Trammell and Walter (1969) in which the individual pulse heights in infinitesimal slices through the detector are summed over the detector thickness. For low trapping, the summed pulse height distribution will be nearly symmetric at low and intermediate energies and we can therefore assume $\Delta E_{\mathrm{c}}$ is normally distributed. For this case, several semi-empirical formulas have been proposed for the summed response. For example, Henck et al. (1970) derived an expression for planar detectors in which $\Delta E_{\mathrm{c}} \propto E^{-1 / 2}$ whereas Owens (1985) predicted that $\Delta E_{\mathrm{c}} \propto E$ for coaxial detectors. Because of the uncertainties in the functional form of $\Delta E_{\mathrm{c}}$ and our imprecise knowledge of $F$, a semi-empirical approach is generally used to describe the resolution function in which

$$
\Delta E=2.355 \sqrt{F \varepsilon E+\left(\Delta E_{\mathrm{e}} / 2.355\right)^{2}+a_{1} E^{a_{2}}} \quad, \quad(\mathrm{keV})
$$

where the electronic noise component $\Delta E_{\mathrm{e}}$ is generally measured directly using a precision pulser and $F, a_{1}$ and $a_{2}$ are treated as semi-empirical constants determined by best-fitting. Eq. (7) is found to fit the resolution functions of compound semiconductors reasonably well with the charge trapping exponent, $a_{2}$, varying between 2 and 3. Recently, the validity of the functional form of the trapping term has been rigorously tested by Kozorezov et al. (2005), who derived a general analytical expression, which includes the effects of geometry. Interestingly, it can be expressed in the form $G(E) E^{2}$, in which the function $G(E)$ reduces to a constant when the $1 / e$ absorption lengths become comparable with the detector thickness - which is invariably true for spectroscopic detectors.

\section{Present detection systems}

Of the fifty or so compounds available, less than half have been investigated as possible detection media. This is illustrated in Table 1, in which the materials highlighted in green are those compounds which have shown a spectroscopic response to X-rays (i.e., $E / \Delta E>1$ ) and those highlighted in red are those that have shown some response to radiation (usually $\mathrm{MeV}$ alpha particles). From the green highlighted compounds, only CdTe, CdZnTe and $\mathrm{HgI}_{2}$ have matured sufficiently to produce commercially viable detection systems and another four are tantalizingly close. We describe these materials below. Their physical and electrical properties are listed in table 2 . 
Cadmium zinc telluride $\left(\mathrm{Cd}_{(1-\mathrm{x})} \mathrm{Zn}_{\mathrm{x}} \mathrm{Te}\right)$ is probably the most widely used compound semi-conductor. It has a cubic, zinc-blende type lattice with atomic numbers close to that of CdTe and a density $\sim 3$ times that of Si. CdTe was originally the focus of experimental study in the 1960's, until it was discovered that the addition of a few percent of zinc to the melt results in an increased band gap as well as the energy of defect formation. This in turn, increases bulk resistivities and reduces the dislocation density, resulting in lower leakage currents and higher temperature operation. Specifically, resistivities of CdZnTe are between one and two orders of magnitude greater than that for CdTe and thus leakage currents are correspondingly lower. Additionally, CdZnTe tends not to suffer from the polarization effects normally associated with CdTe. The best spectral performances are achieved with a zinc fraction of $\sim 10 \%$. Typical FWHM energy resolutions at $59.54 \mathrm{keV}$ are in the $1-2$ $\mathrm{keV}$ range at room temperature and decrease with decreasing temperature to a minimum resolution at $\sim-30^{\circ} \mathrm{C}$ (Owens et al., 2002).

Mercuric iodide $\left(\mathrm{HgI}_{2}\right)$ has been investigated as a room temperature $\mathrm{X}$-and gamma-ray detector since the early 1970's. Its wide band gap in conjunction with the high atomic number of its constituent atoms makes it an attractive material for room temperature operable $\mathrm{X}$ - and particularly gamma-ray spectrometers. In fact, since photoelectric absorption varies as $Z^{5}$, the specific sensitivity of $\mathrm{HgI}_{2}$ is about 10 times greater than that of Ge for energies $>100 \mathrm{keV}$. $\mathrm{HgI}_{2}$ belongs to the family of layered structured, heavy metal iodides. It is a relatively soft material that forms a tetragonal lattice at temperatures below $130^{\circ} \mathrm{C}$. This is known as the alpha phase and the crystals appear red in color with a band gap energy of $2.13 \mathrm{eV}$. At temperatures above $130^{\circ} \mathrm{C}$, $\mathrm{HgI}_{2}$ undergoes a phase transformation to an orthorhombic lattice (beta phase) appearing yellow in colour with a band gap energy of $2.5 \mathrm{eV}$. However, when cooled below $130^{\circ} \mathrm{C}$, the material undergoes a destructive phase transition to alpha- $\mathrm{HgI}_{2}$. This precludes melt growth. The fabrication of a pn-junction is usually not necessary, as dark currents are generally very low. While reported energy resolutions are typically in the $\mathrm{keV}$ region, a series of small planar detectors $\left(5 \mathrm{~mm}^{2}\right.$, cylinder, 200 $\mu \mathrm{m}$ thick) developed by Iwanczyk et al. (1989) have achieved energy resolutions of $\sim 200 \mathrm{eV} \mathrm{FWHM}$ at $5.9 \mathrm{keV}$ at $0^{\circ} \mathrm{C}$.

Gallium arsenide (GaAs) has a simple cubic lattice structure with a band gap sufficiently wide $(1.42 \mathrm{eV})$ to permit room temperature operation but small enough so that its Fano limited spectroscopic resolution is close to that of Si. Compared to other compounds, the performances of GaAs detection systems have steadily advanced, primarily due to improvements in both bulk material properties and detector fabrication techniques. Schottky barrier detectors fabricated using semi-insulating GaAs have consistently demonstrated reasonable room temperature performances. Recently, Owens et al. (2003a) fabricated a series of detectors from ultra-high purity GaAs grown by chemical vapor phase deposition (CVPD). From the I/V characteristics, the typical current densities at $-100 \mathrm{~V}$ were $<0.04 \mathrm{nA} / \mathrm{mm}^{2}$ at room temperature. This is about a factor of 3-100 lower than normally obtained with CVPD detectors and a factor of $10^{3}-10^{4}$ lower than bulk detectors. The measured FWHM energy resolutions of a $0.8 \mathrm{~mm}^{2}, 40 \mu \mathrm{m}$ thick device operated at $-20^{\circ} \mathrm{C}$ were $435 \mathrm{eV}$ FWHM at $5.9 \mathrm{keV}$ and $670 \mathrm{eV} \mathrm{FWHM} \mathrm{at} 59.54 \mathrm{keV}$. At room temperature the corresponding resolutions were $572 \mathrm{eV}$ and $780 \mathrm{eV}$, respectively. Spectral resolutions 
of only $200 \mathrm{eV}$ fwhm at $5.9 \mathrm{keV}$ have been recorded for pixel detectors $(250 \times 250 \times$ $40 \mathrm{~m}^{3}$ ), fabricated from the same base material (Owens et al., 2001a).

Silicon carbide $(\mathrm{SiC})$ is currently being explored as a high temperature $\mathrm{Si}$ alternative that is also chemical and radiation tolerant. It has several distinct advantages over $\mathrm{Si}$, in that it has twice the thermal conductivity and eight times the maximum breakdown field. The former property is important for producing thermally stable or high power semiconductor devices, while the latter means that much higher biases can be applied, resulting in higher drift velocities and better charge collection. Structurally, $\mathrm{SiC}$ is a polymorph with over 200 polytypes possible. However, only three have properties suitable for X-ray detection, namely $3 \mathrm{H}, 4 \mathrm{H}, 6 \mathrm{H}^{1}$ and of these $4 \mathrm{H}$ has the best electrical properties. At present, the X-ray performance of prototype detectors is still relatively poor, with FWHM resolutions of $2.7 \mathrm{keV}$ at $59.54 \mathrm{keV}$ being reported (Bertuccio et al., 2001). However, recent measurements with small pixel detectors have achieved room temperature energy resolutions of $693 \mathrm{eV} \mathrm{FWHM}$ at $59.54 \mathrm{keV}$ (Bertuccio et al., 2003).

Indium phosphide (InP) is direct band gap material whose structural and electronic properties are similar to $\mathrm{Si}$ and GaAs. It has a zinc-blende type crystal structure, with a single non-destructive phase-transition below the melting point making it amenable to standard growth techniques. It has one of the highest electron mobilites of any semiconductor material ( $\sim 3$ times that of silicon) making it particularly suitable for applications where high-count rate operation is desirable (e.g., at synchrotrons). Its band gap of $1.35 \mathrm{eV}$ implies that detectors should operate at room temperature with a Fano limited spectroscopic resolution close to that of Si and its relatively large density, (approximately twice that of $\mathrm{Si}$ ) ensures high X-ray detection efficiency above $10 \mathrm{keV}$. Currently, for small detectors of area $3.1 \mathrm{~mm}^{2}$ and thickness $180 \mu \mathrm{m}, \mathrm{FWHM}$ spectral resolutions of $\sim 2.5 \mathrm{keV}$ and $9.2 \mathrm{keV}$ have been achieved at $5.9 \mathrm{keV}$ and $59.54 \mathrm{keV}$, respectively, at a detector temperature of $-60^{\circ} \mathrm{C}$. At $\mathrm{LN}_{2}$ temperature $\left(-170^{\circ} \mathrm{C}\right)$, these figures improve considerably to $911 \mathrm{eV}$ at 5.9 $\mathrm{keV}$ and $2.5 \mathrm{keV}$ at $59.54 \mathrm{keV}$ (Owens, Peacock and Bavdaz, 2003a).

For gamma-ray applications, there has been some effort to develop the heavier compounds with limited success - particularly lead, bismuth and thallium compounds from period VI of the periodic table. For example, $\mathrm{PbI}_{2}$ has been investigated as a stable alternative to $\mathrm{HgI}_{2}$ since it does not exhibit a destructive phase change between the melting point and room temperature and its density is similar. Unfortunately, while thin detectors yield good room temperature X-ray performance (415 eV FWHM at $5.9 \mathrm{keV}$ and $1.4 \mathrm{keV}$ FWHM at $59.54 \mathrm{keV}$ for a $1 \mathrm{~mm}^{2}$ device; Shah et al., 1996), their transport properties are so poor that detectors thicknesses are limited to $\sim 200$ microns. Therefore, there has been little incentive to develop $\mathrm{PbI}_{2}$ for gamma-ray applications over say $\mathrm{HgI}_{2}$, or even lower $Z$ compounds such as $\mathrm{CdZnTe}$ or GaAs. Better results have been obtained with $\mathrm{TlBr}$, with FWHM room temperature energy resolutions of $1.8 \mathrm{keV}$ at $5.9 \mathrm{keV}$ and $3.3 \mathrm{keV}$ at $59.5 \mathrm{keV}$ recorded for detectors of dimensions $2.8 \times 2.8 \times 0.8 \mathrm{~mm}^{3}$ (Owens et al., 2003b). These resolutions were found to improve with decreasing temperature exhibiting a minimum near $-30^{\circ} \mathrm{C}$. At this temperature the FWHM energy resolutions were $800 \mathrm{eV}$ at $5.9 \mathrm{keV}$ and $2.3 \mathrm{keV}$ at

1The "H" numbers describe the stacking sequence of tetrahedrally bonded SiC bi-layers. 
$59.54 \mathrm{keV}$, respectively. Although these detectors did not suffer from the stability problems normally associated with TlBr detectors (Owens et al., 2001b) they did display polarization effects (i.e., time dependent changes in gain and spectral broadening), which were correlated with large energy depositions per unit time.

Finally in Table 3 we summarize some of the best reported spectral resolutions at soft and hard X-ray wavelengths for each of the above materials.

Table 3. The best energy resolutions achieved with prototype planar detectors. Note, the figures are quoted for the collection of both carriers. Spectral enhancement techniques involving single carrier collection have not been employed. The measurements were carried out under uniform illumination using ${ }^{55} \mathrm{Fe}$ and ${ }^{241} \mathrm{Am}$ radioactive sources. For completeness, we also list the resolutions at room temperature (RT) were possible, since there are many applications in which resolving power is not a primary requirement.

\begin{tabular}{|c|c|c|c|c|}
\hline Material & $\begin{array}{c}\text { Detector size } \\
\text { Area, thickness }\end{array}$ & $\begin{array}{c}\Delta \mathrm{E} @ 5.9 \mathrm{keV} \\
(\mathrm{eV})\end{array}$ & $\begin{array}{c}\Delta \mathrm{E} @ \\
59.5 \mathrm{keV}(\mathrm{eV})\end{array}$ & Reference \\
\hline $\mathrm{Si}$ & $0.8 \mathrm{~mm}^{2}, 500 \mu \mathrm{m}$ & $\begin{array}{l}245 @-15^{\circ} \mathrm{C} \\
750 @+15^{\circ} \mathrm{C}\end{array}$ & $\begin{array}{l}524 \\
800\end{array}$ & Owens et al., (2003a) \\
\hline GaAs & $0.8 \mathrm{~mm}^{2}, 40 \mu \mathrm{m}$ & $\begin{array}{c}450 @-22^{\circ} \mathrm{C} \\
572 @ \mathrm{RT}\end{array}$ & $\begin{array}{l}670 \\
780\end{array}$ & Owens et al., (2003a) \\
\hline GaAs pixel & $250 \times 250 \times 40 \mu \mathrm{m}^{3}$ & $\begin{array}{c}219 @-30^{\circ} \mathrm{C} \\
266 @ \mathrm{RT}\end{array}$ & $\begin{array}{l}468 \\
487\end{array}$ & Owens et al., (2001a) \\
\hline $\begin{array}{l}\mathrm{SiC}-4 \mathrm{H} \\
\end{array}$ & $2 \mathrm{~mm}^{2}, 30 \mu \mathrm{m}$ & & $2700 @ \mathrm{RT}$ & Bertuccio et al., (2001) \\
\hline SiC-4H pixel & $0.3 \mathrm{~mm}^{2}, 50 \mu \mathrm{m}$ & & $693 @ \mathrm{RT}$ & Bertuccio et al., (2003) \\
\hline $\mathrm{InP}$ & 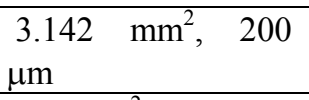 & $\begin{array}{l}911 @-170^{\circ} \mathrm{C} \\
2480 @-60^{\circ} \mathrm{C}\end{array}$ & $\begin{array}{l}3050 \\
9200 \\
\end{array}$ & Owens et al., (2003a) \\
\hline CdTe & $16 \mathrm{~mm}^{2}, 1.2 \mathrm{~mm}$ & $310 @-60^{\circ} \mathrm{C}$ & 600 & Loupilov et al., (2001) \\
\hline CdZnTe & $3.142 \mathrm{~mm}^{2}, 2.5 \mathrm{~mm}$ & $\begin{array}{c}311 @-37^{\circ} \mathrm{C} \\
1508 @ \mathrm{RT}\end{array}$ & $\begin{array}{c}824 \\
2900\end{array}$ & Owens et al., (2002) \\
\hline $\mathrm{HgI}_{2}$ & $5 \mathrm{~mm}^{2}, 200 \mu \mathrm{m}$ & $198 @ 0^{\circ} \mathrm{C}$ & 650 & Iwanczyk et al., (1989) \\
\hline $\mathrm{PbI}_{2}$ & $1 \mathrm{~mm}^{2}, 50 \mu \mathrm{m}$ & $415 @ \mathrm{RT}$ & 1380 & Shah et al., (1996) \\
\hline $\mathrm{TlBr}$ & $3.142 \mathrm{~mm}^{2}, 800 \mu \mathrm{m}$ & $\begin{array}{l}800 @-30^{\circ} \mathrm{C} \\
1800 @ \mathrm{RT}\end{array}$ & $\begin{array}{l}2300 \\
3300\end{array}$ & Owens et al., (2003b) \\
\hline
\end{tabular}

\section{Spectral enhancement techniques}

For conventional radiation detectors fabricated from compound semi-conductors, the wide disparity between the transport properties of the electron and holes, means that detector performances are limited by the carrier with the poorest mobility-lifetime product $(\mu \tau)$. Finite drift lengths introduce an energy dependent depth term into the charge collection process, which effectively limits maximum detector thicknesses to a few $\mathrm{mm}$ for spectroscopy applications. Since mobility is a fundamental material property, the only practical way of improving $\mu \tau$ products is to increase carrier lifetimes, which in turn depends greatly on detector material quality and stoichiometry. In analogy with gas counters, until the particular traps/defect(s) can be identified and corrected, a significant improvement in spectral acuity can be achieved 
using single carrier collection techniques - in other words discarding the carrier with the poorest transport properties - albeit at the expense of detection efficiency. Single carrier sensing can be achieved electronically by pulse rise time discrimination (Jordanov, Prantazis and Huber 1996) and single carrier collection by careful design (e.g., hemispherical; Richter and Siffert 1992) or coplanar grid detectors (Luke 1995) or, in the case of pixel detectors, by exploiting the small-pixel effect (Barrett, Eskin and Barber 1995). In fact, such techniques have been widely applied to CdZnTe detectors to overcome poor hole transport. From Table 2, we note that the $\mu \tau$ product for electrons is usually about 1-2 orders of magnitude greater than that of the holes which means that for present detection systems, the electrons are nearly fully collected for interactions occurring throughout the detector volume and that spectral tailing is almost exclusively due to holes. We discuss two of these techniques below.

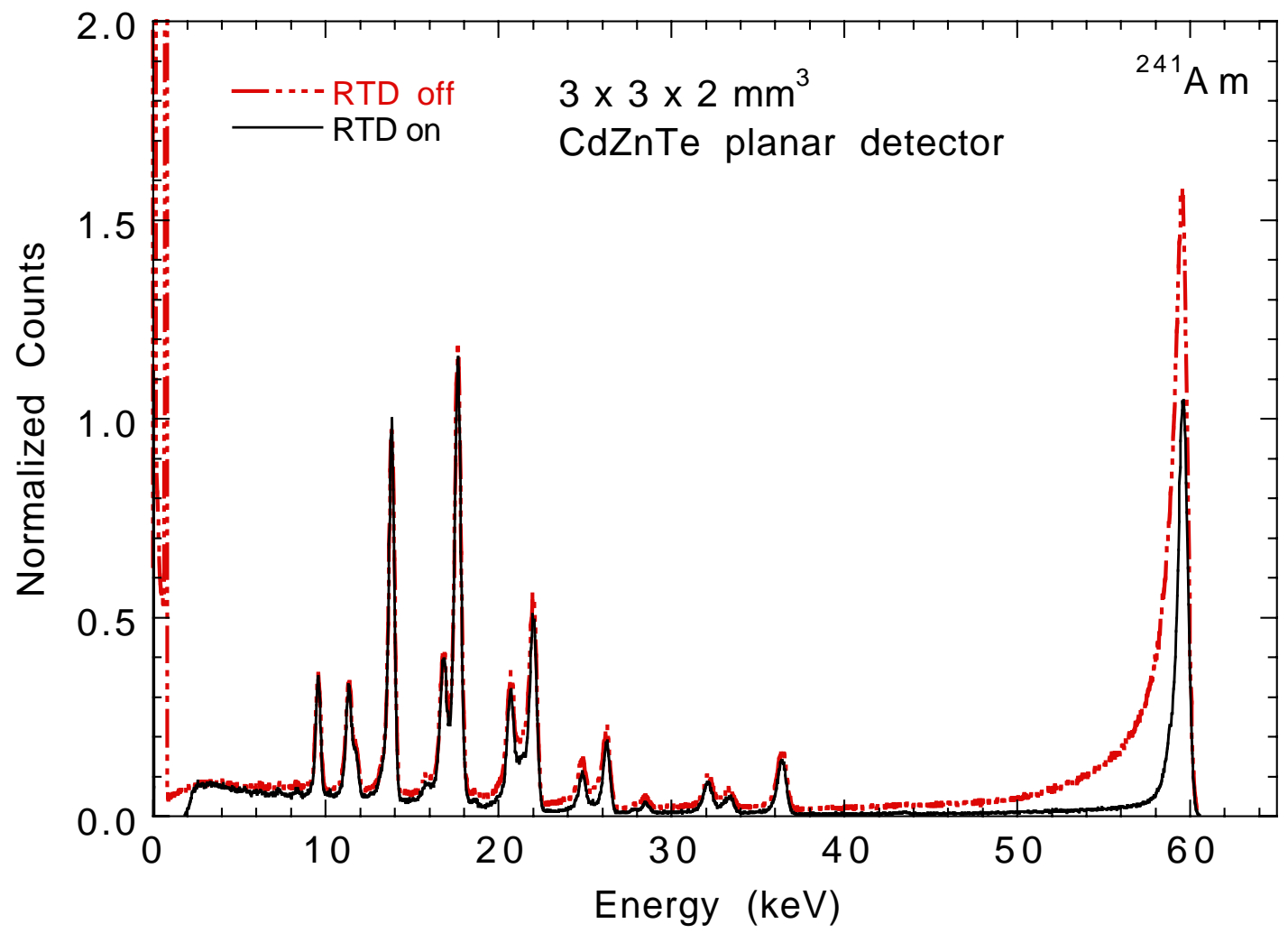

Figure 2. Two ${ }^{241} \mathrm{Am}$ spectra taken with an Amptek $3 \times 3 \times 2 \mathrm{~mm}^{3} \mathrm{CdZnTe}$ detector, illustrating the effectiveness of rise time discrimination (RTD) (Owens et al., 2005a). From the figure we see that the hole tailing is substantially reduced in the case when RTD is used and in fact the FWHM energy resolution improves from $1 \mathrm{keV}$ to $700 \mathrm{eV}$ at $59.54 \mathrm{keV}$. However, an energy dependent decrease in efficiency is also apparent.

\subsection{Rise time discrimination}

Rise time discrimination (RTD) is a relatively simple method (Jordanov, Prantazis and Huber 1996) to improve spectral shape and relies on the fact that for widely different transport properties, the rise times of the current pulses due to the electrons and holes are quite different. With reference to fig. 1, the time taken for an electron and a hole to traverse the detector width $L$ is 
$t_{\mathrm{e}}=\frac{L}{\mu_{\mathrm{e}} E} \quad$ and $\quad t_{\mathrm{h}}=\frac{L}{\mu_{\mathrm{h}} E}$

from which we can see that the electron and hole transit times are directly dependent on carrier mobilities. Consider interactions in the detector volume. Then, since $\mu_{\mathrm{e}}>>$ $\mu_{\mathrm{h}}$, the rising edge of the detector signal will be composed of a fast component due to the electrons and a slow component due to the holes. In rise time discrimination methods, all pulses with a rise time exceeding a pre-set threshold are rejected - in fact all those events that would normally lie in the tail. The effect of rise time discrimination (RTD) is demonstrated in fig. 2, in which we show the measured response of a $3 \times 3 \times 2 \mathrm{~mm}^{3} \mathrm{CdZnTe}$ detector (Owens et al., 2005a). While the resolution is improved (from $1 \mathrm{keV}$ FWHM at $59.54 \mathrm{keV}$ to $700 \mathrm{eV}$ ), using RTD the efficiency is lower than that expected from the physical dimensions of the detector, since many counts are rejected. This is also demonstrated in fig. 2 in which we can see that the amplitude of the lowest peak at $\sim 9 \mathrm{keV}$ is essentially the same in both spectra, since the entire signal is due to the electrons. However, there is an increasing loss of counts in subsequent peaks of the RTD-on spectrum as the energy increases. In fact, the ratio of counts in the $60 \mathrm{keV}$ photopeak (i.e., RTD-on/RTD-off) is $\sim 0.35$ and the ratio of peak heights is $\sim 0.65$.

Since both rise time and signal amplitude are correlated with the depth of interaction, one can use these measurements to deduce charge collection efficiency and thus improve spectroscopic performance. Such methods are termed bi-parametric methods. In practice, it is achieved by plotting rise time as a function of pulse amplitude and energy, from which one can generate a set of correction factors. The measured induced charge is then multiplied by the appropriate correction factor, yielding a corrected pulse amplitude. This technique permits high resolution with little loss of sensitive volume. For example, Verger et al., (2001) report measuring a FWHM energy resolution of $6.5 \%$ at $122 \mathrm{keV}$ using a room temperature $4 \times 4 \times 6$ $\mathrm{mm}^{3} \mathrm{CdZnTe}$ detector with standard planar electrodes. The quoted acceptance efficiency was $>80 \%$. However, there are disadvantages. Firstly, the electronics is complicated and secondly, since the pulse height deficit varies non-linearly with rise time, the technique is only valid over a limited detector depth. Systems have been developed that apply non-linear corrections and effectively use the entire depth of the detector. However, their complexity and the difficulty of calibration, make them uncommercial at this time.

\subsection{Co-planar grid detectors}

The co-planar grid technique (Luke 1995) is essentially an evolution of the classical Frisch grid, introduced to reduce position dependent charge collection effects in gas detectors. By including a grid within the gas volume near the anode of the device, it is possible to bias the electrodes such that electrons are drifted towards the anode through the grid and positive ions are drifted in the opposite direction. According to the Shockley-Ramo theorem, the induced charge on the anode is primarily from electrons moving from the grid to the anode, hence the photon interaction position dependence of the pulse shape is greatly reduced. A similar concept has been shown to work with semiconductor devices - coplanar grid devices. The anode electrodes take the form of inter-digitated grids that are connected to 
separate charge-sensitive preamplifiers. An electric field is established in the detector bulk by applying bias to the cathode, which is a full-area contact located on the side opposite the grid electrodes. The two grid preamplifiers are connected to a subtraction circuit to produce a difference signal. Bias is applied between the grid electrodes so that one of the grids preferentially collects charge. Charge motion within the bulk of the detector is sensed equally by the grid electrodes and the difference is essentially zero. However, when charge approaches the anode, the grid signals begin to differ and a signal is registered at the output of the difference circuit. In a well-designed detector and in the absence of electron trapping, the magnitude of the difference signal is the same no matter where the charge is generated in the device. This results in a large improvement in performance for gamma-ray spectroscopy when compared to conventional planar device technology. In fact, operating the grids as a simple planar detector results in no spectroscopic signal, but when operated in the co-planar geometry, the results are quite dramatic and are illustrated in fig. 3 (from Owens et al., 2005b).

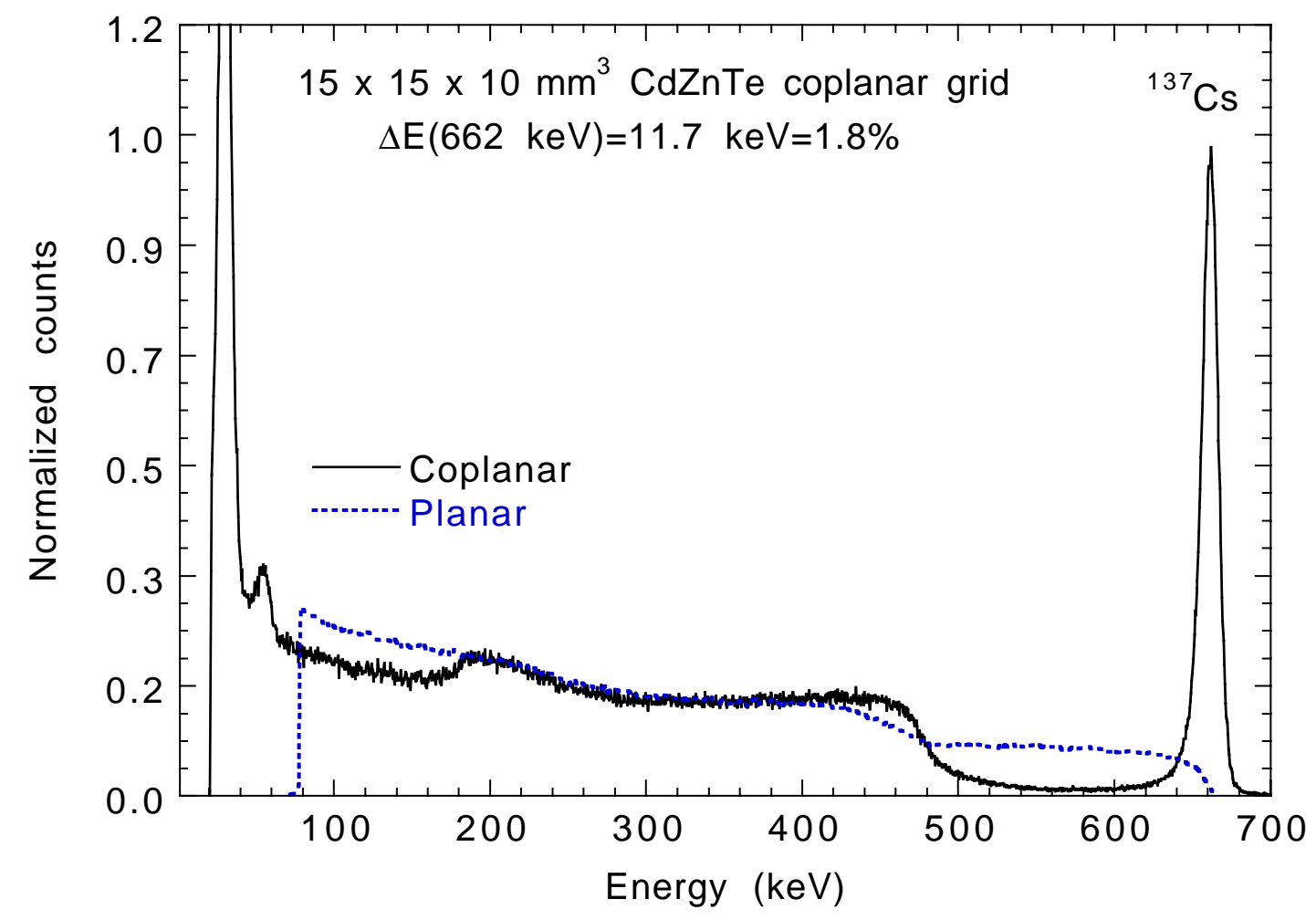

Figure 3. Comparison of pulse height spectra measured with a $2.25 \mathrm{~cm}^{3}$ device $(15 \mathrm{~mm} \times 15 \mathrm{~mm} \times 10$ $\mathrm{mm}$ ) - when operated as a planar detector and as a coplanar grid detector (Owens et al., 2005b).

\section{Discussion and Conclusions}

While novel designs can improve energy resolution, ultimately carrier transport still limits the maximum detection volume. For the transport properties appropriate for $\mathrm{CdZnTe}$, this is about $10 \mathrm{~cm}^{3}$. Thus, ultimately the quality of the material has to be improved if a substantial increase in detector volume is to be achieved - sufficient to produce an effective gamma-ray detector. In the longer term, material improvements must be paralleled by corresponding developments in heterostructure and quantum 
heterostructure technology, provided of course strain is not a limiting factor. Heterostructures may solve the problem of contacting by building up a series of semiconductor layers until it is possible to satisfy the relationship that the workfunction of the metal contact is less that that of the semiconductor (i.e., $\phi_{\mathrm{m}}<\phi_{\mathrm{s}}$ ) for n-type material and greater than that of the semiconductor for $\mathrm{p}$-type material. At the present time, the first purpose-built heterostructures for X-ray applications are being reported in the literature (Silenas et al. 2003).

\section{References}

Barrett, H., Eskin, J., Barber, H. (1995). Phys. Rev. Letts., 75, 156 - 159.

Bertuccio, G., Casiraghi, R., Nava, F. (2001). IEEE Trans. Nucl. Sci., NS-48, $232-$ 233.

Bertuccio, G., Casiraghi, R., Gatti, E., Maiocchi, D., Nava, F., Canali, C., Cetronio, A., Lanzieri, C. (2003). Materials Science Forum, vols 433-436, 941-944

Hecht, K. (1932). Z. Physik, 77, 235-245.

Henck, R., Gutknecht, D., Siffert, P., De Laet, L., Shoenmaekers, W. (1970). IEEE Trans. Nucl. Sci., NS-17, 149-159.

Iwanczyk, S., Yang, Y.J., Bradley, J.G., Conley, J.M., Albee, A.L., Economou, T.E. (1989). IEEE Trans. Nucl. Sci., NS-36, 841-845.

Iwanczyk, J.S., Schnepple, W.F., Masterson, M.J. (1992). Nucl. Instr. and Meth., A322, 421-426.

Jordanov, V.T., Pantazis, J.A.,. Huber, A.C. (1996). Nucl. Instr. and Meth., A380, 353-357.

Kozorezov, A., Wigmore, K., Owens, A., den Hartog, R., Peacock, A., Al-Jawhari, H.A. (2005). Nucl. Instr. and Meth., A546, 209-212.

Loupilov, L., Sokolov, A., Gostilo, V. (2001). Rad. Phys. and Chem., 61, 463-464.

Luke, P.N. (1995). IEEE Trans. Nucl. Sci., NS-42, 207-213.

Owens, A. (1985). Nucl. Instr. and Meth., A238, 473-478.

Owens, A., Bavdaz, M., Peacock, A., Poelaert, A., Andersson, H., Nenonen, S., Tröger, L., Bertuccio, G. (2001a). J. App. Phys., 90, 5376-5381.

Owens, A., Bavdaz, M., Lisjutun, I., Peacock, A., Zatoloka, S. (2001b). Nucl. Instr. and Meth., A458, 413-417.

Owens, A., Bavdaz, M., Andersson, H., Gagliardi, T., Krumrey, M., Nenonen, S., Peacock, A., Taylor, I. (2002). Nucl. Instr. \& Meth., A484, 242-250.

Owens, A., Peacock, A., Bavdaz, M. (2003a). Proc. of the SPIE, 4851, 1059-1070.

Owens, A., Bavdaz, M., Brammertz, G., Gostilo, V., Graafsma, H., Krumrey, M., Lisjutin, I., Peacock, A., Puig, A., Sipila, H., Zatoloka S. (2003b). Nucl. Instr. and Meth., A497, 370-380.

Owens, A., Buslaps, T., Erd, C., Graafsma, H., Hijmering, R., Lumb, D., Welter, E. (2005a). Nucl. Instr. and Meth., in press.

Owens, A., Buslaps, T., Gostilo, V., Graafsma, H., Hijmering, R., Kozorezov, A., Loupilov, A., Lumb, D., Welter, E. (2005b). Nucl. Instr. and Meth., in press.

Ramo, S. (1939). Proc. IRE, 27, 584-585.

Richter, M., Siffert, P. (1992). Nucl. Instr. and Meth., A322, 529-537. 
Shah, K., Olschner, F., Moy, L., Bennett, P., Misra, M., Zhang, J., Squillante, M., Lund, J. (1996). Nucl. Instr. and Meth., A380, 266-270.

Shockley, W. (1938). J. Appl. Phys., 9, 635-636.

Silenas, S., Pozela, K., Dapkus, L., Jasutis, V., Juciene, V., Pozela, J., Smith, K. (2003). Nucl. Instr. and Meth., A509, 30-33.

Trammell, R., Walter, F.J. (1969). Nucl. Instr. and Meth., 76, 317-321.

Verger, L., Boitel, M., Gentet, M.C., Hamelin, R., Mestais, C., Mongellaz, F., Rustique, J., Sanchez, G. (2001). Nucl. Instr. and Meth., A458, 297-309. 